\title{
Selective targeting of microglia by quantum dots
}

\author{
S Sakura Minami ${ }^{1,2}$, Binggui Sun ${ }^{1,2}$, Ketul Popat ${ }^{3,5}$, Tiina Kauppinen ${ }^{2}$, Mike Pleiss', Yungui Zhou', Michael E Ward ${ }^{1,2}$, \\ Paul Floreancig ${ }^{4}$, Lennart Mucke ${ }^{1,2}$, Tejal Desai ${ }^{3}$ and Li Gan ${ }^{1,2^{*}}$
}

\begin{abstract}
Background: Microglia, the resident immune cells of the brain, have been implicated in brain injury and various neurological disorders. However, their precise roles in different pathophysiological situations remain enigmatic and may range from detrimental to protective. Targeting the delivery of biologically active compounds to microglia could help elucidate these roles and facilitate the therapeutic modulation of microglial functions in neurological diseases.
\end{abstract}

Methods: Here we employ primary cell cultures and stereotaxic injections into mouse brain to investigate the cell type specific localization of semiconductor quantum dots (QDs) in vitro and in vivo. Two potential receptors for QDs are identified using pharmacological inhibitors and neutralizing antibodies.

Results: In mixed primary cortical cultures, QDs were selectively taken up by microglia; this uptake was decreased by inhibitors of clathrin-dependent endocytosis, implicating the endosomal pathway as the major route of entry for QDs into microglia. Furthermore, inhibiting mannose receptors and macrophage scavenger receptors blocked the uptake of QDs by microglia, indicating that QD uptake occurs through microglia-specific receptor endocytosis. When injected into the brain, QDs were taken up primarily by microglia and with high efficiency. In primary cortical cultures, QDs conjugated to the toxin saporin depleted microglia in mixed primary cortical cultures, protecting neurons in these cultures against amyloid beta-induced neurotoxicity.

Conclusions: These findings demonstrate that QDs can be used to specifically label and modulate microglia in primary cortical cultures and in brain and may allow for the selective delivery of therapeutic agents to these cells.

\section{Background}

Chronic inflammation is a hallmark of many neurological diseases [1-5]. Microglia, innate immune cells of the CNS, become activated in response to injury and appear to have important roles in the defense against invading microbes and in wound repair [6]. They also phagocytose dead cells and help clear misfolded protein aggregates, such as those formed by amyloid beta $(A \beta)$ in Alzheimer's disease (AD) [7]. However, under certain pathophysiological circumstances, microglia may also contribute to neuronal toxicity. For example, factors released from activated microglia can amplify inflammatory processes that contribute to neurodegeneration [8]. To harness and modulate the activity of microglia, it would be useful to be able to target biologically active compounds specifically to these powerful cells.

\footnotetext{
* Correspondence: Igan@gladstone.ucsf.edu

'Gladstone Institute of Neurological Disease, 1650 Owens St., San Francisco CA 94158, USA

Full list of author information is available at the end of the article
}

Previously, we used viral vectors and a microglia-specific promoter to selectively modulate gene expression in microglia [9]. However, the usefulness of this approach is limited by the possibility of inflammatory responses, potential toxicity associated with viral infections, and the inability of viral vectors to deliver a variety of chemical compounds. Here, we demonstrate that quantum dots (QDs) can effectively deliver biologically active molecules to microglia in vitro and in vivo.

Semiconductor fluorescent QDs are nanometer-sized particles with unique optical and electrical properties that make them particularly suited for visualization and tracking of living cells [10-12]. They have a heavy metal core, consisting for instance of cadmium and selenium or cadmium and tellurium, and an unreactive zinc sulfide shell. Manipulation of the core size allows synthesis of a wide array of QDs emitting at various wavelengths, visible as different colors. Because of their composition and small size, these nanoparticles are readily excitable by light and display minimal photobleaching [13]. Importantly, the
Ciomed Central

(c) 2012 Minami et al; licensee BioMed Central Ltd. This is an Open Access article distributed under the terms of the Creative Commons Attribution License (http://creativecommons.org/licenses/by/2.0), which permits unrestricted use, distribution, and reproduction in any medium, provided the original work is properly cited. 
outer coating can be modified to allow for the attachment of different bioactive molecules, offering unprecedented possibilities to visualize and modulate molecular processes in living cells $[14,15]$. QDs have been used for molecular imaging in diverse biological systems [16]. In most cases, surface-immobilized antibodies or peptides were used to direct QDs to specific cellular targets. For example, QDs conjugated to nerve growth factor (NGF) effectively activate TrkA receptors and downstream signaling cascades that promote neuronal differentiation [17]. QDs not conjugated to specific antibodies or peptides appear to have limited ability to enter most cells, especially at low concentrations [18]. Unconjugated QDs were found to be localized to macrophages and microglia that infiltrate experimental gliomas [19]. However, whether QDs are selectively taken up by microglia under normal conditions is unknown. Here we examined the ability of QDs to enter microglia in primary cultures and mouse brains and the underlying cellular mechanisms.

\section{Methods}

\section{Quantification of QD uptake}

Water-soluble ZnS capped CdSe streptavidin coated quantum dots (QDs) with emission at $655 \mathrm{~nm}$ were purchased from Invitrogen. QD solution was added to mixed cortical cultures at $0.5 \mathrm{nM}$ for 1-48 h. The uptake of QDs was visualized under epifluorescence or confocal microscopy with an XF02-2 filter from Omega Optical that allows simultaneous multi-color viewing (Omega Optical, Brattleboro, VT). For visualization of QD655 uptake in mouse brain, confocal images were taken with a Nipkow spinning disk confocal microscope. GFP signal was imaged with a $488 \mathrm{~nm}$ laser and $515 \mathrm{~nm}$ bandpass, and QDs were imaged with a $405 \mathrm{~nm}$ and a $700 \mathrm{~nm}$ bandpass emission filter. Images were acquired in $0.5 \mathrm{~nm}$ step sizes in the $\mathrm{z}$ dimension. The amounts of QDs taken up by the cells were quantified with MetaMorph (Molecular Devices Corporation, Sunnyvale, CA). To investigate the mechanisms by which microglia take up QDs, cortical cultures were pretreated with chlorpromazine (CPZ) (6-20 $\mu \mathrm{M})$, cytochalasin B (CTB) (4-8 $\mu \mathrm{M})$, bafilomycin (BAF) (25-50 nM), mannan (1-2 $\mathrm{mg} / \mathrm{ml})$, polyinosinic acid (100-200 $\mu \mathrm{g} / \mathrm{ml})$, or blocking antibodies (anti-mannose receptor, antimacrophage scavenger receptor, $2 \mu \mathrm{M}$ ) for $2 \mathrm{~h}$ before adding QD solutions, followed by $24 \mathrm{~h}$ incubation before analyses.

\section{Primary mixed culture and microglial culture}

Cortices were isolated from Sprague-Dawley rat pups (Charles River Laboratories, Wilmington, MA) on postnatal day 0 or 1 . To establish mixed cortical cultures, cells were plated at 160,000 cells $/ \mathrm{ml}$ in plating medium containing Dulbecco's modified Eagle's medium (DMEM), 10\% fetal bovine serum, $0.5 \mathrm{mM}$ glutamax, and
$100 \mathrm{U} / \mathrm{ml}$ penicillin and $100 \mu \mathrm{g} / \mathrm{ml}$ streptomycin for 7 days, as described [9].

Primary microglial cultures were prepared from 1-dayold mice as described [20]. Cortices were dissociated by mincing and incubation in papain and DNase. After centrifugation for $5 \mathrm{~min}$ at $500 \times g$, the cells were resuspended by trituration with a fire-polished Pasteur pipette, plated on 6-well plates (Falcon/BD Biosciences) at a density of $6.4 \times 10^{5}$ cells/well, and maintained in a $37^{\circ} \mathrm{C}, 5 \%$ $\mathrm{CO}_{2}$ incubator. The culture medium consisted of Eagle's minimal essential medium (MEM, Gibco) supplemented with $10 \%$ endotoxin-free fetal bovine serum (HyClone), $2 \mathrm{mM}$ glutamine, and streptomycin. After 2 weeks in vitro, microglia were harvested by mildly shaking the cultures and collecting the floating cells. These cells were re-plated at a density of $5 \times 10^{5}$ cells/well in 24-well plates. The microglial cultures were used for experiments 2 days after re-plating. Each culture well was visually inspected by phase contrast microscopy before use, and wells containing contaminating astrocytes or greater than $30 \%$ activated microglia were excluded. Microglia with enlarged soma and less than two branching processes were considered activated. Experiments were performed in MEM, in which all drug stocks were diluted. All tissue culture supplies were purchased from Invitrogen (Carlsbad, CA) unless stated otherwise.

\section{Cytokine assay}

Microglial cultures were placed in $300 \mu \mathrm{l}$ MEM alone (ctrl) or with QDs $(0.5 \mathrm{nM})$ for $6 \mathrm{~h}$, after which the Qdots were washed out, and the microglia were placed in fresh MEM or lipopolysaccharide (LPS) $(50 \mathrm{ng} / \mathrm{ml})$ for 20-22 h. Medium samples were taken and treated with complete protease inhibitor and stored in $-70^{\circ} \mathrm{C}$. Medium $(50 \mu \mathrm{l})$ was evaluated with a Beadlyte mouse 14-plex cytokine detection system (Millipore), according to the manufacturer's instructions. This immunoassay method employs 14 cytokine-specific antibodies tagged with fluorescent beads. Assays were performed in duplicate, and the fluorescent signal corresponding to each cytokine was measured with a BioPlex 200 system (Biorad). Values were normalized to the protein content of each well as determined by the bicinchoninic assay [21].

\section{Immunocytochemistry}

Cultures were fixed in $4 \%$ paraformaldehyde in phosphatebuffered saline (PBS) for 15-30 min at room temperature. After permeabilization in PBS with $0.1 \%$ Triton for $10 \mathrm{~min}$, cells were placed in blocking buffer (PBS with $10 \%$ FBS and $0.01 \%$ Triton) for $30 \mathrm{~min}$. To label microglia, primary antibodies to Iba-1 (1:250, Wako Pure Chemical Industries, Osaka, Japan) or anti-CD11b (1:200, Chemicon, Temecula, CA) were applied in blocking buffer overnight 
at $4^{\circ} \mathrm{C}$ and visualized with anti-rabbit (Iba-1) or anti-rat (CD11b) conjugated with FITC (Vector Laboratories, Burlingame, CA). To identify astroglia or neurons, GFAP (1:1000; DAKO, Carpinteria, CA) or MAP2 (1:500; Chemicon, Temecula, CA) antibodies, respectively, were applied in blocking buffer for $2 \mathrm{~h}$ at room temperature or overnight at $4^{\circ} \mathrm{C}$ and visualized with anti-rabbit (GFAP) or anti-mouse (MAP2) conjugated with FITC (Vector Laboratories).

\section{Stereotaxic injection of QDs into the brain}

The QD solution (3 $\mu \mathrm{l}$ at $100 \mathrm{nM}$ ) was stereotaxically injected into the hippocampus of $C X 3 C R^{+/-}$mice, which express green fluorescent protein (GFP) in microglia, at the following coordinates relative to bregma: anterior posterior: -2.1 , medial lateral: \pm 1.7 , dorsal ventral: -2.0 . The brains were perfused and fixed in $4 \%$ paraformaldehyde 2-28 days later.

\section{Quantum dots conjugation with saporin}

The avidin-biotin affinity interaction was used to conjugate saporin to QDs. Briefly, $2 \mu \mathrm{l}$ of QDs $(1 \mu \mathrm{M})$ were mixed with $2 \mu \mathrm{l}$ of biotinlyated saporin $(56 \mu \mathrm{M})$, and $76 \mu$ of PBS was added. The solutions were incubated at room temperature with continuous shaking for at least $2 \mathrm{~h}$ before they were used for analysis or treatment. X-ray photoelectron spectroscopy (XPS) analysis was performed to determine the chemical composition of saporin-conjugated QDs. Unconjugated and saporin-conjugated QDs were adsorbed on a silicon surface for ease with XPS analysis. An x-ray photoelectron spectrometer with a monochromatic Al-Ko-Xray small spot source $(1486.6 \mathrm{eV})$ and multichannel detector was used for this analysis. A concentric hemispherical analyzer was operated in constant analyzer transmission mode to measure the binding energies of emitted photoelectrons. The binding energy scale was calibrated by the Au4f7/2 peak at $83.9 \mathrm{eV}$, and the linearity was verified by the $\mathrm{Cu} 3 p 1 / 2$ and $\mathrm{Cu} 2 p 3 / 2$ peaks at 76.5 and $932.5 \mathrm{eV}$, respectively. Survey spectra were collected from 0 to $1100 \mathrm{eV}$ with pass energy of 160 $\mathrm{eV}$, and high-resolution spectra were collected for the $\mathrm{C} 1 s$ peak with pass energy of $10 \mathrm{eV}$. All spectra were referenced by setting the $\mathrm{C} 1 s$ peak to $285.2 \mathrm{eV}$ to compensate for residual charging effects. Data for percent atomic composition and atomic ratios were calculated using analysis software. For peak fit analysis, a convolution of Gaussian components was assumed for all peak shapes. High-resolution C1s scans were taken to further support the presence of saporin on the QDs. The major hydrocarbon peak $(\mathrm{C}-\mathrm{C})$ is at $285.2 \mathrm{eV}$. The binding energy at $286.8 \mathrm{eV}$ is assigned to amines $\left(\mathrm{CH}_{2} \mathrm{~N}\right)$ and the binding energy at $288.0 \mathrm{eV}$ is assigned to amide functional groups $(\mathrm{O}=\mathrm{C}-\mathrm{N})$.

\section{$A \beta$ treatment and cell death quantification}

A $\beta$ 1-42 peptides lyophilized in hydroxyfluroisopropanol (HFIP) were purchased from rPeptide (Athens, GA). Lyophilized $A \beta$ powder was reconstituted immediately before being diluted in Neurobasal A/N2 medium to 10-20 $\mu \mathrm{M}$. Loss of neurons in mixed cultures was measured as described [9]. MAP2-positive neurons were counted in 15-40 random fields under a fluorescence microscope $(400 \times$ magnification $)$.

\section{Results}

Selective uptake of QDs by microglia in primary cortical cultures

The passive uptake of QDs was investigated in primary cultures derived from neonatal rat cortices, which contain three main cell types: MAP2-positive neurons, GFAP-positive astrocytes, and CD11b-positive microglia [9]. Commercial QDs (QD-streptavidin, Invitrogen; emitting at $655 \mathrm{~nm}$ ) were applied to the mixed culture system at different concentrations and visualized 1-48 h later. QDs were selectively internalized by microglia labeled with antibodies against CD11b or Iba-1 (Figure 1A). At QD concentrations of $0.5 \mathrm{nM}, 38.9 \pm 4.5 \%$ of Iba- 1 -positive microglia contained QDs after $18 \mathrm{~h}$ incubation. In contrast, no QDs were observed in GFAP-positive astroglia (Figure 1B) or MAP-2 positive neurons (Figure $1 \mathrm{C}$ ). At $4 \mathrm{nM}$ concentration, microglia started to internalize QDs within $1 \mathrm{~h}$ (Figure 1D), followed by a gradual intracellular accumulation of QDs during the next $48 \mathrm{~h}$ (Figure 1D). These results suggest that QDs are stable and not sensitive to intracellular degradation. Importantly, after $72 \mathrm{~h}$ incubation, QDs did not colocalize with Annexin V/Sytox green, which labels apopotic/necrotic cells (Figure 1E), suggesting that uptake and retention of QDs does not induce toxicity in culture.

To determine whether the selective uptake of QDs by microglia is influenced by surface chemistry, we used QDs with an amine-derivatized polyethylene glycol (PEG) outer coating that reacts directly with amine-reactive groups (QD655-PEG), QDs with a carboxyl coating (QD655-carboxy), or QDs with a PEG coating conjugated to streptavidin (QD655-PEG-Strep). QD655-PEG, QD655-PEG-Strep, and QD655-Carboxy were all selectively taken up by microglia in mixed cortical cultures (Figure 1F).

We next tested whether the size of QDs affected their ability to enter microglia. QDs of different sizes emit at different wavelengths of the visible spectrum; therefore, QDs of different sizes can be distinguished by their color. We compared QDs emitting at 525, 605, 655, or $705 \mathrm{~nm}$, and found that QD655 was the most efficient at entering microglia (Figure 2A-B). However, despite their different efficiencies, all QDs tested were selectively taken up by microglia in mixed cortical cultures, regardless of their emission wavelengths. 


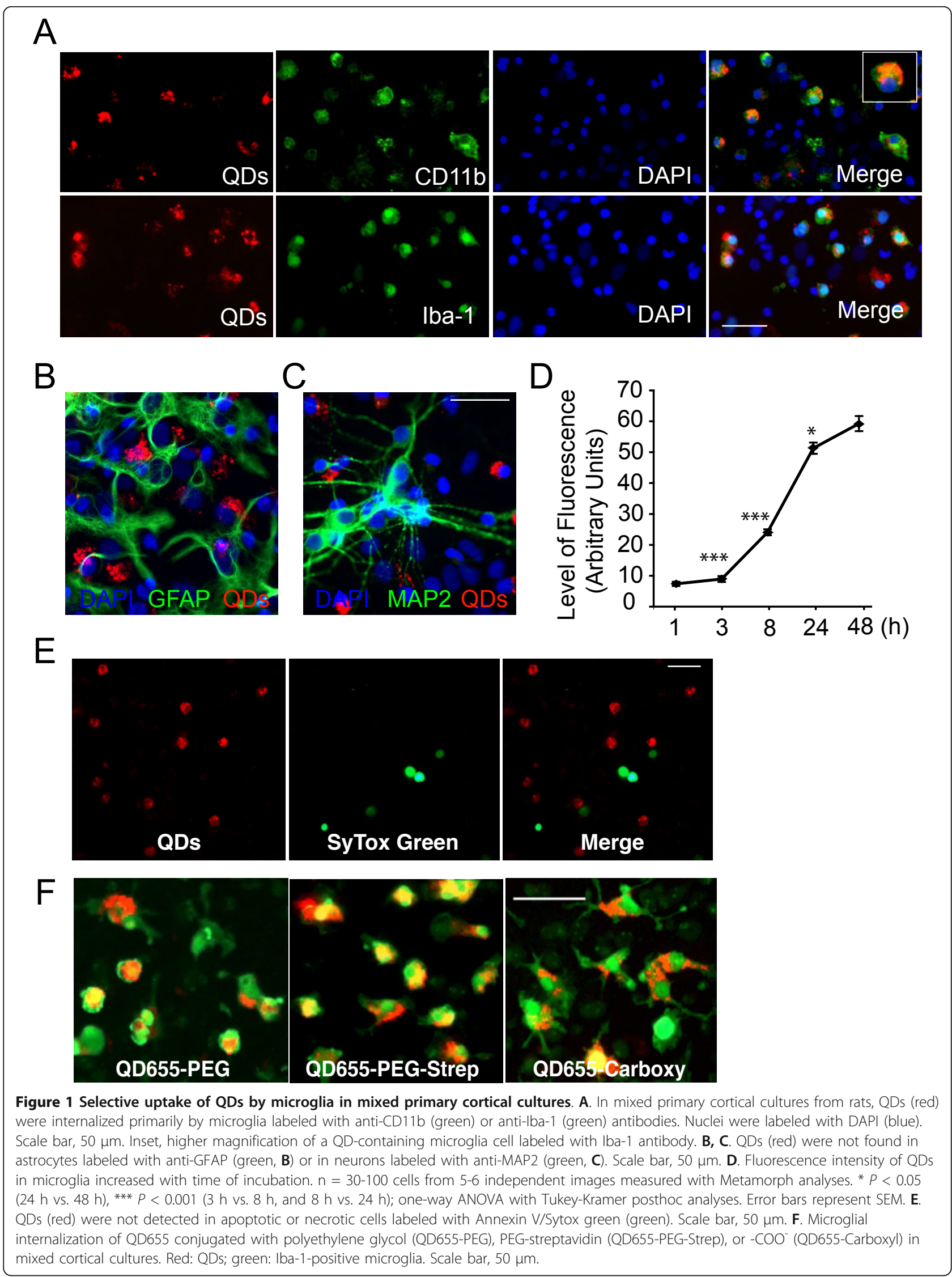




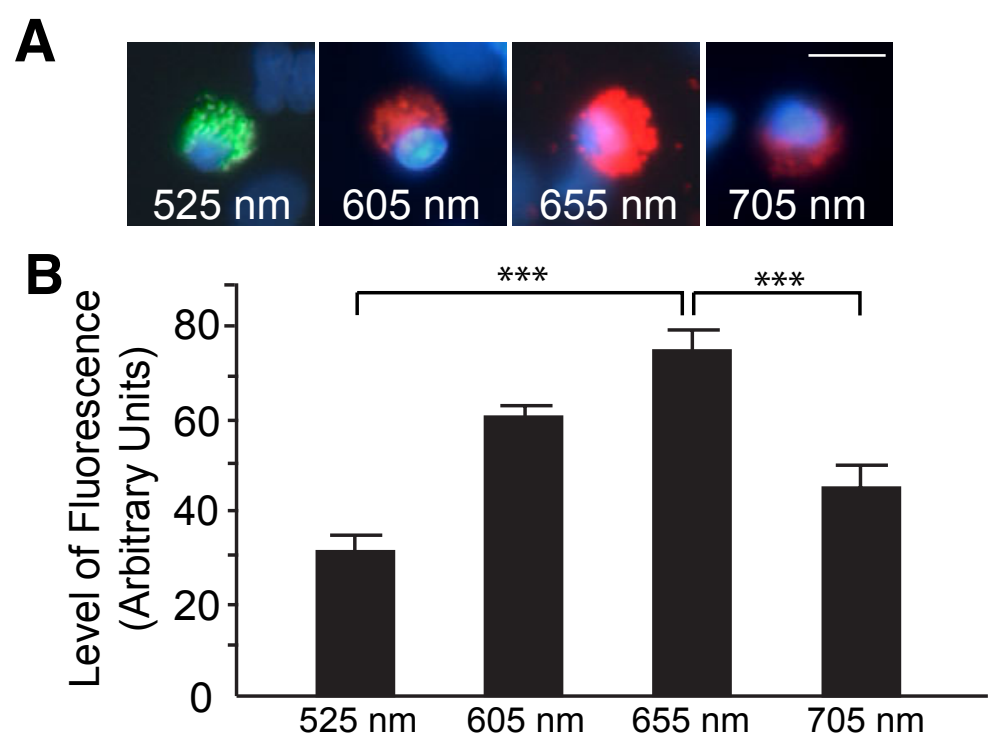

Figure 2 Size of QDs affects their uptake by microglia. A. Internalization of QDs of different sizes by microglia. Blue: DAPI. B. Size-dependent internalization of QDs by microglia was quantified by fluorescence intensity with Metamorph analyses. $n=23-39$ cells from 5-6 independent images. ${ }^{* *}, P<0.001$ by Tukey-Kramer posthoc test. Bars represent mean \pm SEM. Scale bar, $20 \mu \mathrm{m}$.

QD uptake does not affect release of cytokines in primary microglia

To determine if uptake of QDs per se alters microglial function, we measured the release of inflammatory cytokines from primary microglia treated with QDs or with medium alone. QD treatment of primary microglia did not alter their release of tumor necrosis factor $\alpha$ (TNF $\alpha$ ), KC, RANTES, MIP-1 $\alpha$, MIP-1 $\beta$, or IP-10 (Table 1). Treatment with QDs also did not change the microglial release of cytokines in response to lipopolysaccharide (Table 1).

The uptake of QDs by microglia depends on clathrinmediated endocytosis

We next investigated the mechanism underlying the entry of QDs into microglia. Because the diameter of QDs ranges from 10-100 $\mathrm{nm}$, we hypothesized that QDs traverse the cell membrane via endocytosis rather than phagocytosis [22]. Since pH sensitivity is considered a good indicator of entry by endocytosis, we examined if the uptake of QDs by microglia depends on $\mathrm{pH}$. The specific inhibitor of endosomal proton-ATP pumps bafilomycin (BAF) elevates the $\mathrm{pH}$ in endocytic compartments to neutrality. The entry of QDs into microglia was blocked by BAF in a dose-dependent manner (Figure 3A-B), suggesting that this process depends on the acidification of endosomes.

To further dissect the molecular mechanisms underlying QD endocytosis, we investigated if the entry of QDs depends specifically on clathrin-mediated endocytosis, a well-studied mode of endocytosis that is crucial for

Table 1 Microglial release of cytokines

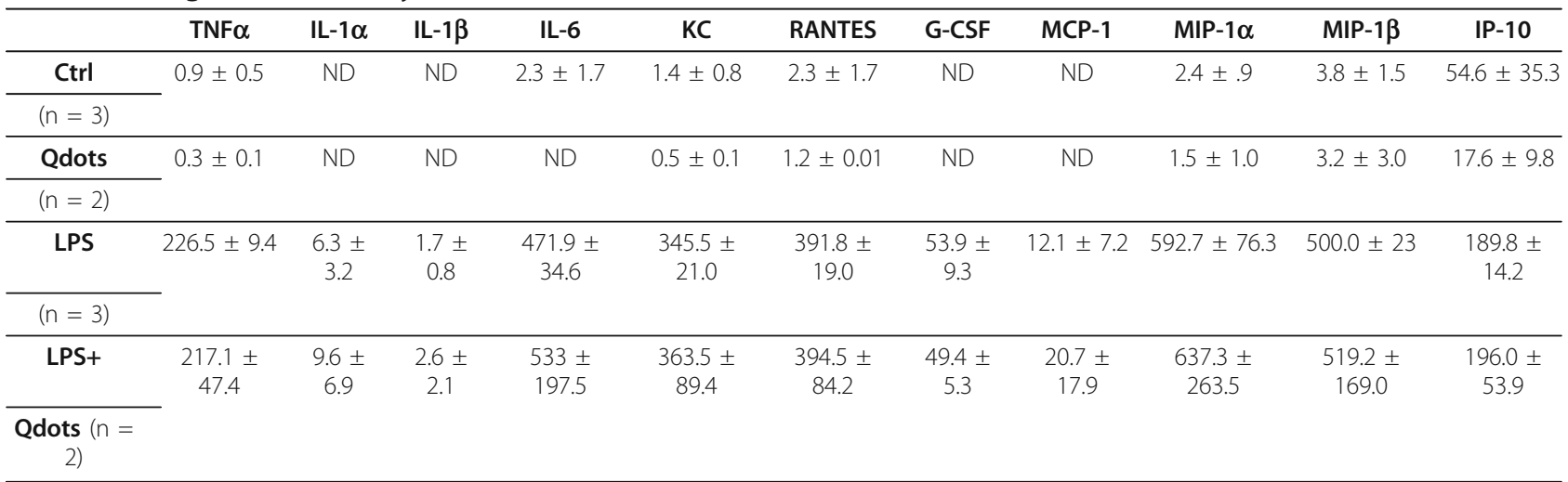

Cytokines $(\mathrm{pg} / \mathrm{ml})$ released by primary microglia treated with vehicle, QD, LPS, or LPS + QD (mean \pm SEM). $\mathrm{n}$, number of wells per condition. ND not detected, GCSF granulocyte colony-stimulating factor, IP-10 $10 \mathrm{kDa}$ interferon-gamma-induced protein, $K C$, keratinocyte chemoattractant, $M C P$ - 1 monocyte chemotactic protein-1, MIP-1 $\alpha$ macrophage inflammatory protein 1 alpha, MIP-1 $\beta$ macrophage inflammatory protein 1 beta, RANTES, regulated upon activation. The following cytokines were undetectable in all conditions: VEGF, IFN $\gamma$, and IL-10 


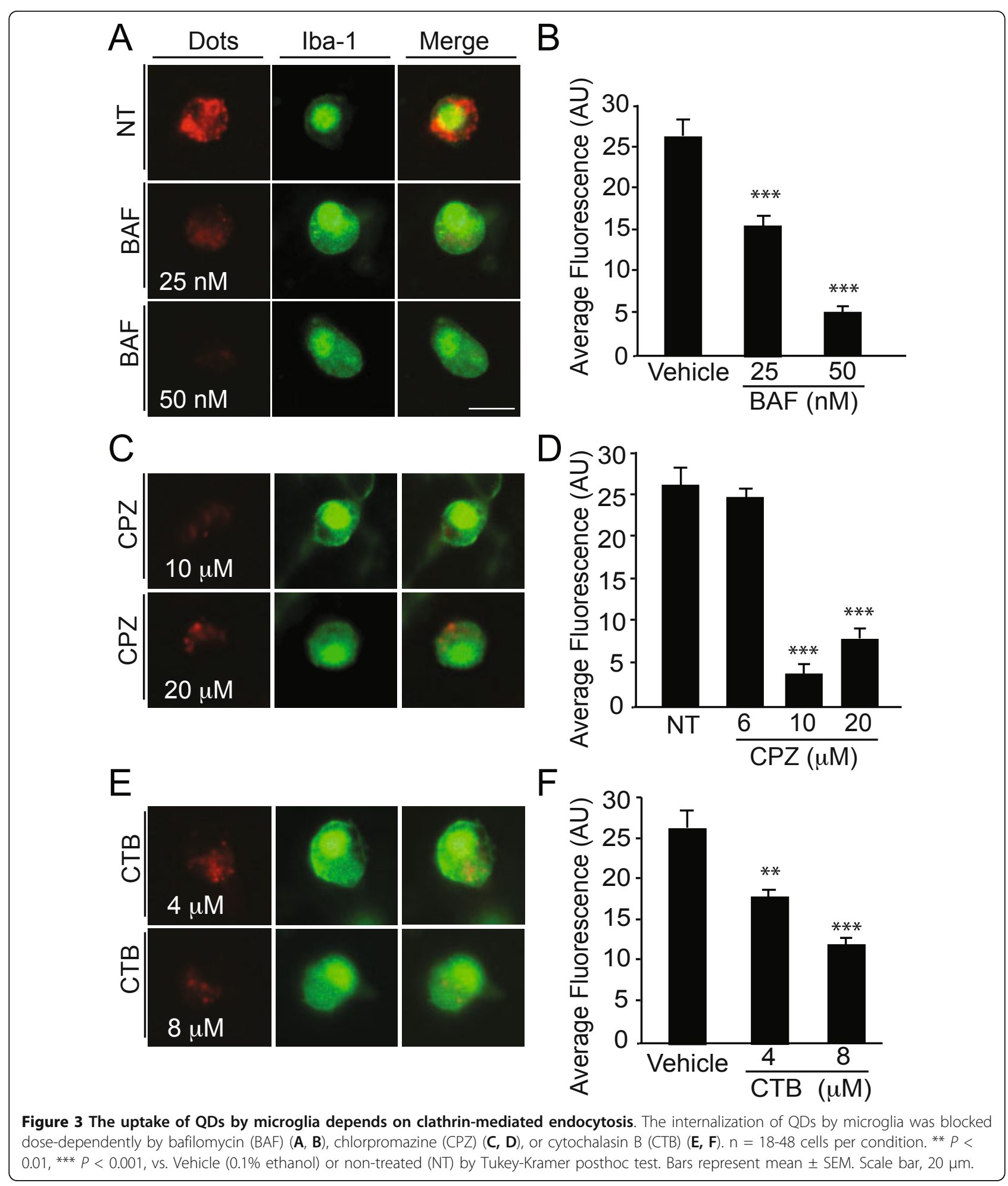

many physiological functions, including the rapid clearance and down regulation of activated signaling receptors and the efficient recycling of synaptic vesicle membrane proteins after neurotransmission. Cells were treated with CPZ, a cationic amphiphilic drug that prevents the recycling of clathrin and thus prevents endocytosis by clathrin-dependent mechanisms [23]. Treatment with CPZ at 10-20 $\mu \mathrm{M}$ significantly inhibited the entry of QDs into microglia (Figure 3C-D). Clathrinmediated entry can be also be inhibited by CTB, which 
induces depolymerization of actin filaments [24]. F-actin dynamics have been shown to be necessary for various stages of clathrin-coated vesicle formation, including coated pit formation, constriction, and internalization [25]. Treatment with CTB induced a dose-dependent decrease in QD entry into microglia (Figure 3E-F). Both $\mathrm{CPZ}$ and $\mathrm{CTB}$ inhibit transferrin (a specific maker for clathrin-mediated endocytosis) at the concentrations used in the current study [26], strongly supporting the notion that QDs enter microglia through clathrinmediated endocytosis.

The uptake of QDs by microglia occurs through mannose and macrophage scavenger receptors

To determine if microglia-specific receptors, such as macrophage scavenger receptor 1 (MSR-1) [27-29] and mannose receptor [30], mediate the uptake of QDs, we treated primary mixed cortical cultures with inhibitors against these receptors and measured QD uptake by microglia. Mannan, an inhibitor of mannose receptor, dose-dependently decreased microglial uptake of QDs (Figure 4A). Polyinosinic acid (PIA), an inhibitor of macrophage scavenger receptor, also decreased microglial uptake of QDs (Figure 4B). Treatment with anti-mannose receptor (MR) or anti-macrophage scavenger receptor (MSR) antibodies also blocked microglial uptake of QDs (Figure $4 C)$. These results implicate microglia-specific receptors in the selective uptake of QDs by microglia.

Selective uptake of QDs by microglia in mouse brains We next injected QDs (3 $\mu \mathrm{l}$ of $100 \mathrm{nM})$ into the hippocampus of $C X 3 C R^{+/-}$mice, which express green fluorescent protein (GFP) in microglia. QDs spread throughout most of the hippocampus (Figure 5A), Consistent with the selective targeting of QDs to microglia in cortical primary cultures, QDs were also predominantly localized in microglia in the brain (Figure 5C-D). Internalized QDs in microglia were further confirmed with Z-stack images (Figure $5 \mathrm{E}$ ) and a $3 \mathrm{D}$ reconstruction of the confocal images (Additional file 1). In contrast, very little uptake of QDs was observed by GFAP-positive astroglia (Figure 5F) or MAP-2-positive neurons (Figure 5G). On some occasions, though, weak fluorescent signals were detected in the neurons of the dentate gyrus, suggesting limited neuronal uptake of QDs at high concentrations in vivo. Our results indicate that in the mouse brains, QDs target microglia preferentially and with high efficiency. Interestingly, the strong fluorescent signal remained stable for at least 1 month after the injection (Figure 6), supporting the feasibility of following the QDs long term.

\section{QD-saporin-mediated depletion of microglia decreases $A \beta$-induced neuronal loss}

We next investigated if QDs could be used to deliver biologically active compounds selectively to microglia. Exposure of mixed cortical cultures to pathogenic A $\beta$ aggregates, which are widely thought to cause AD, results in the degeneration of neurons. Notably, this neurotoxicity is at least partially dependent on the presence of microglia [31,32]. We therefore wanted to determine if the $A \beta$-induced neurotoxicity in such cultures could be suppressed by delivering a cytotoxin specifically to microglia through QDs. For this purpose, the cytotoxin saporin, which belongs to a family of single-
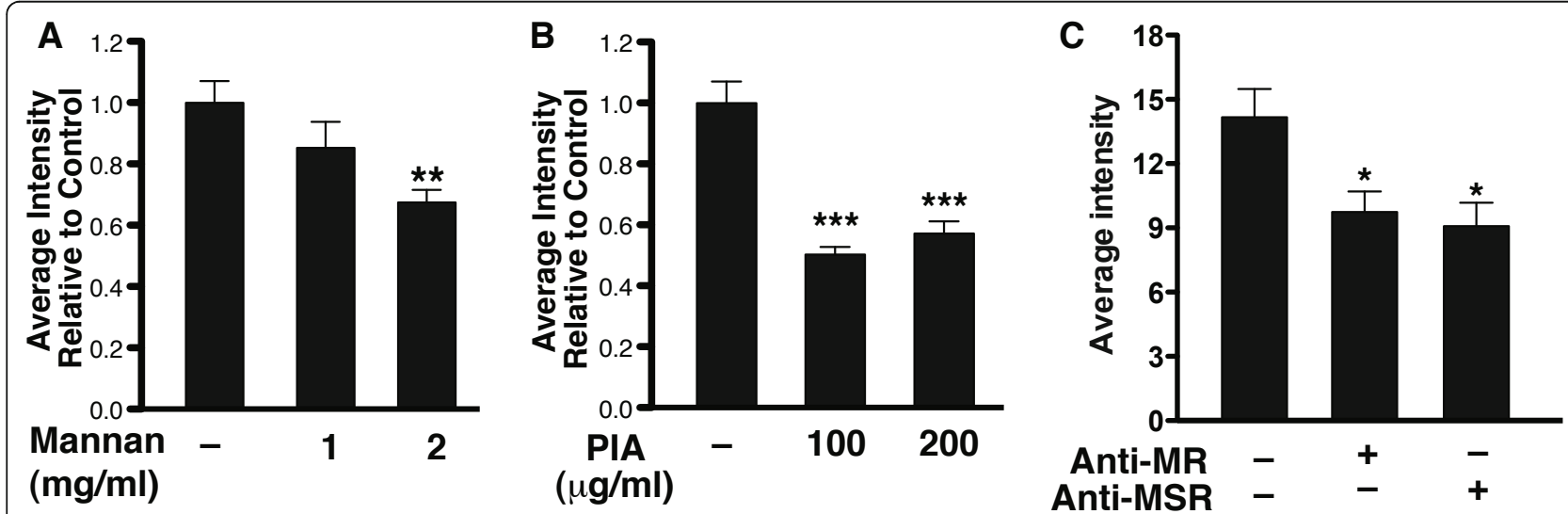

Figure 4 Microglial uptake of QDs occurs through mannose receptors and macrophage scavenger receptors. A. The internalization of QDs by microglia in rat primary mixed cortical cultures was quantified by fluorescence intensity with Metamorph software. Microglial uptake of QDs was blocked in a dose-dependent manner by mannan, an inhibitor of mannose receptors (A) and by polyinosinic acid (PIA), an inhibitor of scavenger receptors (B). $n=120-200$ cells from at least eight separate images. Experiments were repeated three times. $\mathbf{C}$. Antibodies against mannose receptor (MR) or macrophage scavenger receptor (MSR) were applied to rat primary mixed cortical cultures at a $2 \mu \mathrm{M}$ concentration for $2 \mathrm{~h}$ before incubation with QDs. Microglial uptake of QDs was blocked by antibodies against MR and MSR. $\mathrm{n}=105-120$ cells from at least eight separate images. Experiments were repeated twice. ${ }^{* *} P<0.001,{ }^{* *} P<0.01,{ }^{*} P<0.05$ vs. control-treated, one-way ANOVA with TukeyKramer posthoc analyses. Bars represent mean \pm SEM 


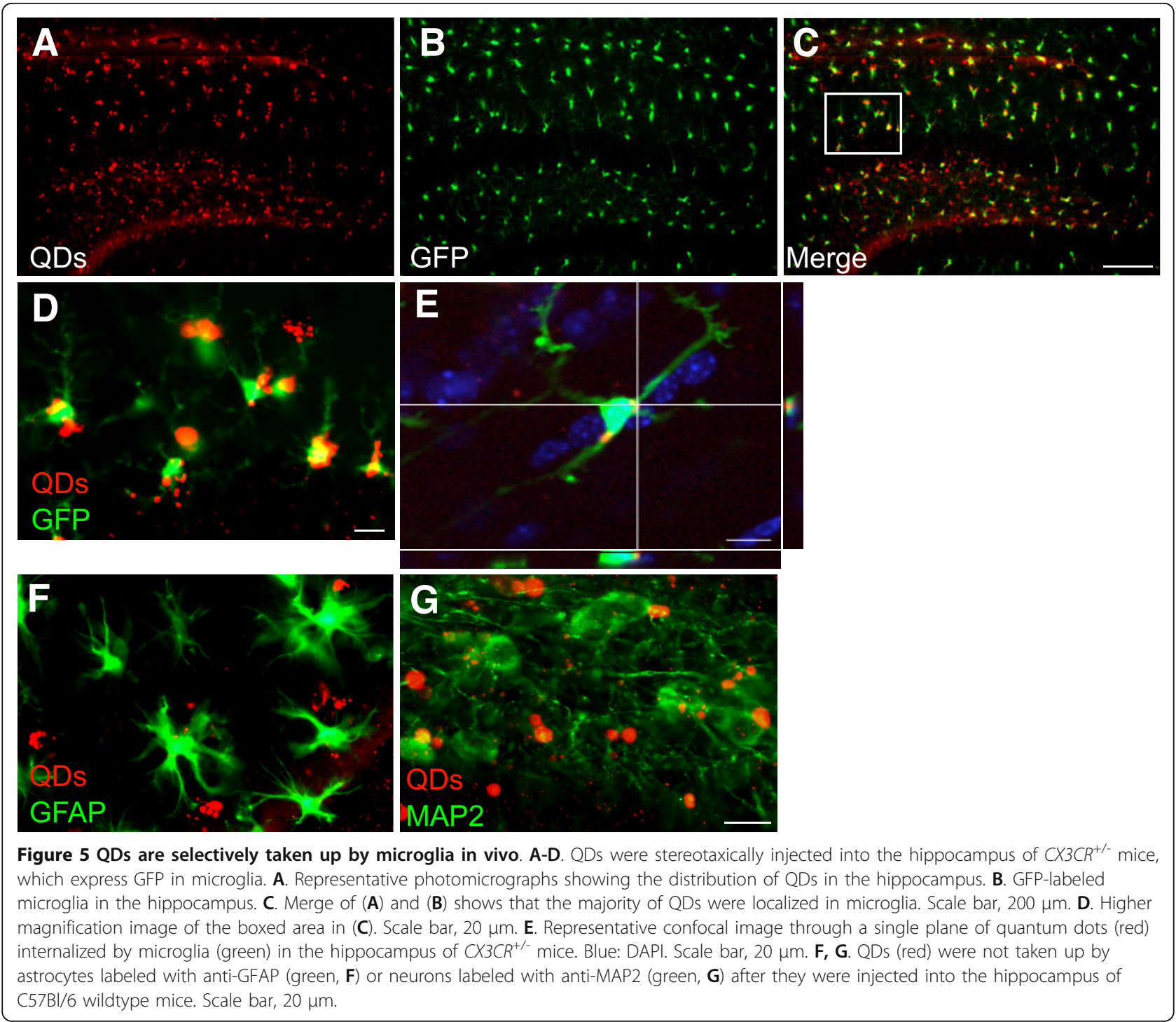

chain ribosome inactivating proteins (RIPs), was biotinylated for coupling with QD-streptavidin. Saporin conjugated to an antibody against Mac1 (CD11b) has been used to selectively kill microglia in hippocampal slice cultures and in rat brain $[33,34]$. Here, we tested whether conjugation of saporin with QDs could result in similar microglia-specific cell loss.

To confirm the conjugation of saporin with QDs, XPS was used to compare the chemical composition of unconjugated and saporin-conjugated QDs adsorbed on a silicon surface. While negligible carbon was present on the silicon surface due to impurities, the carbon composition increased for the silicon surface adsorbed with QDs. There was a further increase in carbon for surface adsorbed with saporin-conjugated QDs, consistent with the presence of streptavidin and saporin, which are composed predominantly of carbon (Figure 7A, Table 2A).
This increase was associated with a decrease in oxygen composition on the surfaces (Figure 7A, Table 2A). Moreover, there was a decrease in the elements present in QDs, namely Se, S, Cd and Zn after conjugation with saporin, further suggesting successful conjugation.

High-resolution $\mathrm{C} 1 \mathrm{~s}$ scans were taken to further support the presence of saporin on the QDs (Figure 5B, Table 2B). The major hydrocarbon peak $(\mathrm{C}-\mathrm{H})$ was at $285.2 \mathrm{eV}$. A binding energy at $286.8 \mathrm{eV}$ is assigned to amines $\left(\mathrm{CH}_{2} \mathrm{~N}\right)$, and a binding energy at $288.0 \mathrm{eV}$ is assigned to amide functional groups $(\mathrm{O}=\mathrm{C}-\mathrm{N})$. For the silicon surface, there was only one peak at $285.2 \mathrm{eV}$. For QDs, apart from the hydrocarbon peak at $285.2 \mathrm{eV}$, peaks for amine (at 286.8 $\mathrm{eV}$ ) and amide (at $288 \mathrm{eV}$ ) were also present. After saporin conjugation, the intensities of the amine and amide peaks increased, as shown in the deconvolution of high-resolution $\mathrm{C} 1 \mathrm{~s}$ spectra into individual peaks. The decreased 

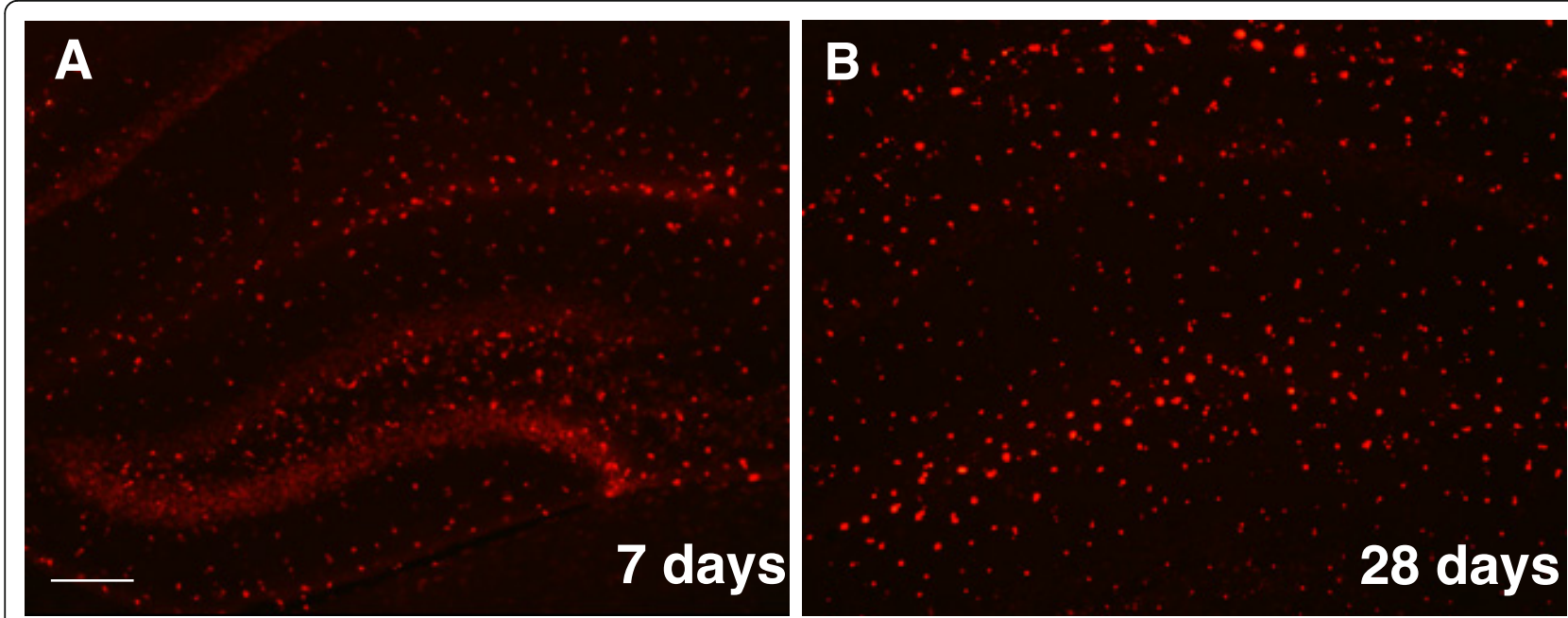

Figure 6 Long-term expression of QDs. A, B. QDs are observed 7 days (A) and 28 days (B) after injection into the hippocampus of adult C57BL/6 mice. Scale bar, $200 \mu \mathrm{m}$.

contribution of the hydrocarbon peak at $285.2 \mathrm{eV}$ and the subsequent increase in amide and amine peaks at other binding energies reflect the successful conjugation of the QD surface with saporin.

We then applied QDs conjugated with saporin to primary cultures for 2 days and quantified microglia after the treatment by Iba-1 expression. Application of QD-Sap caused a marked reduction of microglia labeled with an antibody against Iba-1, without affecting the number or morphology of neurons or astroglia (Figure 8A and 8B). Treatment with unconjugated QDs or saporin alone did not significantly affect the number of microglia compared with untreated control (data not shown).
Next, we tested the effects of QD-Sap-induced microglial ablation on $\mathrm{A} \beta$ toxicity in mixed cortical cultures. Depletion of microglia with QD-Sap significantly increased the number of MAP2-positive neurons that survived A $\beta 1$ 42 treatment (Figure $8 \mathrm{C}$ ). Application of QDs or saporin alone had no effects. Thus, neurons could be preserved in this neurodegenerative disease model through the selective targeting of microglia with modified QDs.

\section{Discussion}

Our study shows that QDs are preferentially taken up by microglia in mixed cortical cultures and in brain. We further showed that the major cellular uptake pathway
A

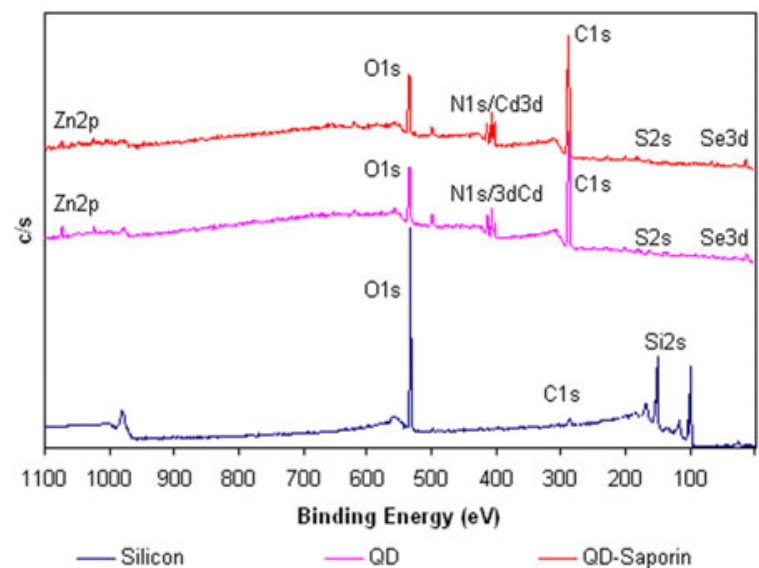

B

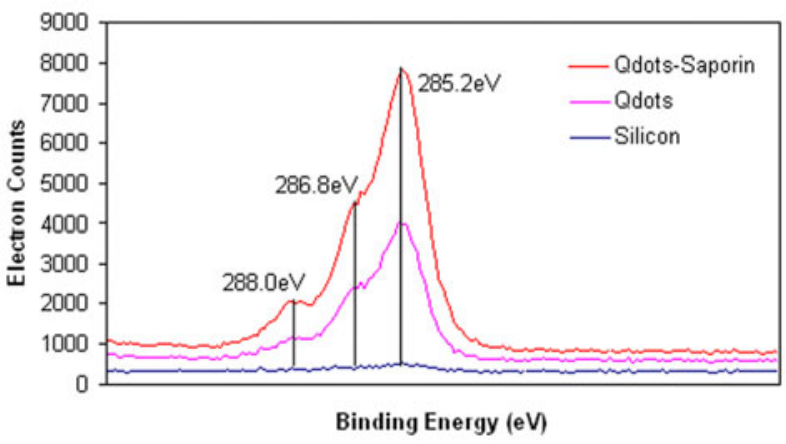

Figure 7 Conjugation of QDs with saporin by streptavidin-biotin binding. A. Comparison of carbon composition of QDs and QD-saporin conjugates measured by X-ray photoelectron spectroscopy. B. High-resolution C1 scans confirmed the presence of saporin on the surface of QDs. 
Table 2 Saporin conjugation of QDs characterized by Xray photoelectron spectroscopy

\begin{tabular}{|c|c|c|c|c|c|}
\hline \multirow[b]{2}{*}{ A. } & & \multirow[t]{2}{*}{ Binding Energy $(\mathrm{eV})$} & \multicolumn{3}{|c|}{ Atomic \% } \\
\hline & & & Silicon & QDs & QD-Sap \\
\hline & $\mathrm{Se}_{3 / 2}$ & 57 & 0 & 0.47 & 0.36 \\
\hline & $\mathrm{Si} 2 \mathrm{~s}$ & 151 & 50.62 & 0 & 0 \\
\hline & $\mathrm{S} 2 \mathrm{p}_{1 / 2}$ & 165 & 0 & 1.8 & 1.25 \\
\hline & $\mathrm{C} 1 \mathrm{~s}$ & 285 & 9.09 & 78.08 & 80.27 \\
\hline & N1s & 398 & 0 & 1.24 & 0.74 \\
\hline & $\mathrm{Cd} 3 \mathrm{~d}_{5 / 2}$ & 405 & & & \\
\hline & O1s & 523 & 40.29 & 17.94 & 17.02 \\
\hline & $\mathrm{Zn} 2 \mathrm{p}_{3 / 2}$ & 1022 & 0 & 0.47 & 0.36 \\
\hline B. & & & Silicon & QDs & QD-Sap \\
\hline & $285.2 \mathrm{eV}$ & C-H (hydrocarbon) & 100 & 65 & 54 \\
\hline & $286.8 \mathrm{eV}$ & $\mathrm{CH}_{2} \mathrm{~N}$ (amine) & 0 & 27 & 32 \\
\hline & $288.0 \mathrm{eV}$ & $\mathrm{O}=\mathrm{C}-\mathrm{N}$ (amide) & 0 & 8 & 14 \\
\hline
\end{tabular}

A. Surface elemental composition calculated by XPS survey scans. B. Highresolution $\mathrm{C} 1 \mathrm{~s}$ peak deconvoluted into its components and indicating the composition of individual peaks on each surface

of QDs in microglia is clathrin-mediated endocytosis involving the microglia-specific receptors MSR-1 and mannose receptor. In primary mixed cortical cultures, QDs effectively delivered the cytotoxin saporin selectively to microglia. Depletion of microglia with QD-Sap resulted in protection against microglia-mediated $A \beta$ toxicity.

Our finding that QDs are selectively taken up by microglia is consistent with previous observations that QDs were localized to macrophages and microglia that infiltrate experimental gliomas [19]. However, in contrast to the previous study, which suggested that QDs were phagocytosed by macrophages and microglia, our data indicate that QDs enter microglia via receptor binding and clathrin-mediated endocytosis. In eukaryotes, macromolecules enter the cell in membranebound vesicles either via 'phagocytosis' (the uptake of particles larger than $0.5 \mu \mathrm{m}$ in diameter) or 'pinocytosis' (the uptake of fluid and solutes) [22]. Phagocytosis occurs by an actin-dependent mechanism and is usually independent of $\mathrm{pH}$ gradient and clathrin, whereas pinocytosis occurs by at least four basic mechanisms: macropinocytosis, clathrin-mediated endocytosis, calveolaemediated endocytosis, and clathrin- and calveolae-independent endocytosis [22]. Interestingly, the uptake of soluble $A \beta$ by microglia was found to be mediated through a nonsaturable, fluid phase macropinocytic mechanism that is distinct from phagocytosis and receptor-mediated endocytosis [35]. The size of QDs, which range from $10-100 \mathrm{~nm}$ in diameter, makes it unlikely that QDs enter microglia via phagocytosis. Indeed, the blockade of QD entry by balifomycin, chlorpromazine, and cytochalasin B provide strong evidence that QDs are taken up by microglia via clathrin-mediated endocytosis. Clathrin-mediated endocytosis occurs in all cell types. However, blocking the MSR-1 or mannose receptor with specific inhibitors or antibodies prevented the uptake of QDs, indicating that the selective targeting of QDs to microglia requires their binding to microgliaspecific receptors. We cannot exclude that other microglial receptors such as Fc-receptors, complement receptors, and Toll-like receptors might also mediate the endocytosis of QDs by microglia. Additional studies are needed to fully characterize the potential binding sites of QDs on the microglial surface.

The unique optical properties of quantum dots, such as high quantum yields, large molar extinction coefficients, size-dependent tunable emission and high photostability, make them appealing as fluorescent probes for biological imaging. On the other hand, because of their size range, QDs are also very suitable for manipulations at the molecular level, offering new approaches for the delivery of potent bioactive agents. Microglia may have roles in the pathogenesis of various CNS diseases, including multiple sclerosis, Alzheimer's disease, Parkinson's disease, and amyotropic lateral sclerosis [36-39]. Our finding that certain sizes of QDs selectively target microglia provides a novel platform to probe and modulate biological processes in microglia and may lay the foundation for the development of QD-based reagents that can modulate specific signaling pathways in microglia.

In contemplating the therapeutic potential of QDs, an important caveat is their biocompatibility and toxicity $[18,40]$. The use of PEG on the surface of the QDs significantly improved their biocompatibility and minimized their toxicity [41-43]. A gene profiling study showed that application of high-dose QDs only induced changes in a small number of genes associated with the transport machinery, supporting the feasibility of longterm usage of QDs in biological systems [41]. The current study provides evidence that targeting of microglia with QDs is unlikely to result in toxicity through increased cytokine release, even in the presence of LPSstimulated microglial activation. Indeed, our data suggest that the toxicity of QDs is limited, at least in the short term. However, evidence suggests that QDs may activate autophagy, implicating an important role in the regulation of normal cell processes [44-47]. The sizedependent induction of autophagy by QDs could result in the initiation of a cell death cascade [48]. Alternatively, the induction of autophagy during inflammation may protect against the harmful effects of microglial activation. Further investigation will be required to establish the long-term effects of the material, especially the heavy-metal component, in biological systems. If 

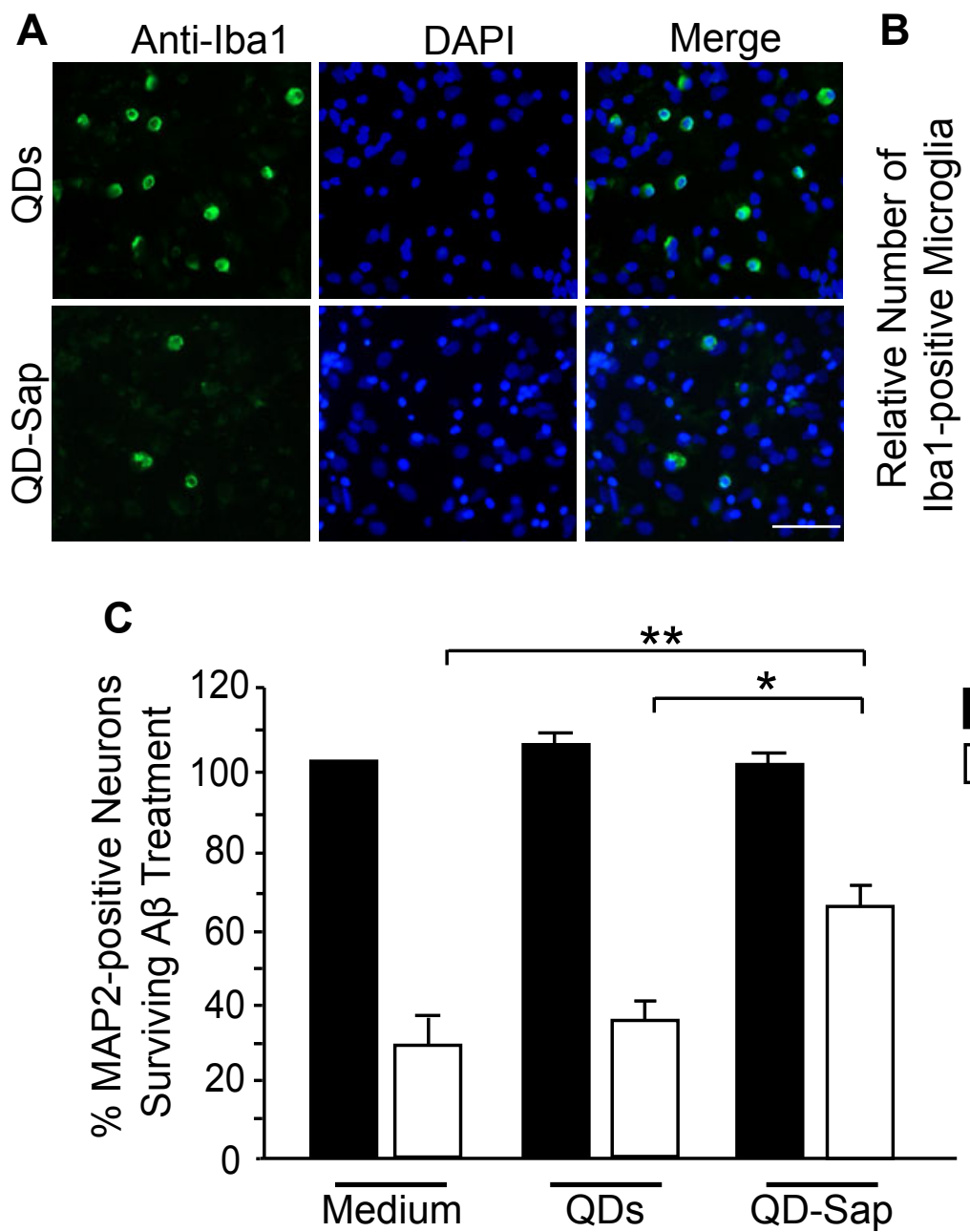

B

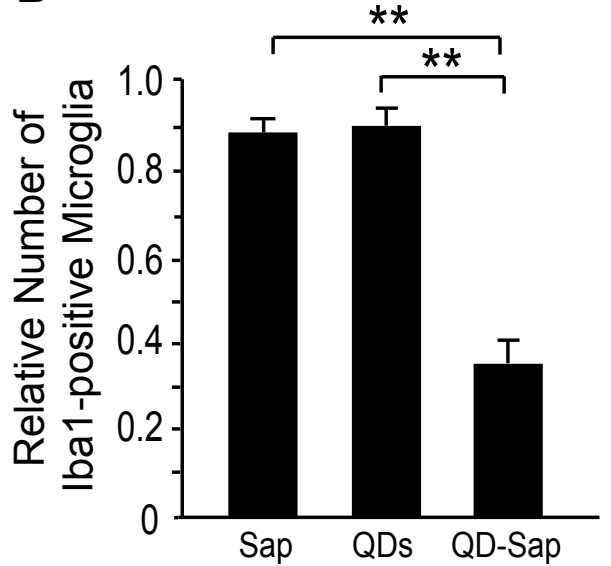

Untreated $\mathrm{A} \beta 1-42$

Figure 8 QD-saporin-mediated depletion of microglia decreases neuronal loss in mixed cortical cultures exposed to $A \beta$. $A$. Representative photomicrographs of microglial cells in mixed cortical cultures treated with QDs or QD-saporin (QD-Sap) conjugates. Microglial cells were labeled with anti-lba-1 (green) and nuclei were labeled with DAPI (blue). B. Quantification of Iba-1-positive cells in mixed cultures treated with unconjugated saporin (Sap), QDs, or QD-saporin conjugates (QD-Sap). C. Quantification of MAP2-positive neurons in mixed cultures treated with A 1 1-42 after pretreatment with medium alone, QDs, or QD-Sap. $\mathrm{n}=5$ wells from three independent experiments. ${ }^{*} P<0.05$, ${ }^{* *} P<$ 0.01, one-way ANOVA with Tukey-Kramer posthoc analyses. Scale bar, $100 \mu \mathrm{m}$.

their safety profile continues to improve, QDs may emerge as a novel approach for the selective delivery of therapeutic agents to microglia in diverse CNS diseases.

\section{Conclusions}

In conclusion, our study demonstrates that QDs can be used to specifically label and modulate microglia in primary cortical cultures and in the brain. This specificity is in part due to the selective uptake by macrophage scavenger receptors and mannose receptors present on the surface of microglia. These findings may allow for the selective imaging and delivery of therapeutic agents to microglia in a wide range of neurological disease models and, ultimately, perhaps also in the corresponding human conditions.

\section{Additional material}

Additional file 1: Quantum dots are internalized by microglia. $C X 3 C R$ +/ mice, which express GFP in microglia, were injected with QD655 into the hippocampus. QD were imaged $48 \mathrm{~h}$ after injection, and z-stack confocal images were compiled into a 3D movie. Red: QDs, Green: microglia.

\section{Abbreviations}

AD: Alzheimer's disease; BAF: balifomycin; CNS: central nervous system; CPZ: chlorpromazine; CTB: cytochalasin B; G-CSF: granulocyte colony-stimulating factor; GFAP: glial fibrillary acidic protein; GFP: green fluorescent protein; IFNץ: interferon-gamma; IL-10: interleukin 10; IP-10: 10-kDa interferongamma-induced protein; KC: keratinocyte chemoattractant; LPS: lipopolysaccharide; MAP2: microtubule-associated protein 2; MCP-1: monocyte chemotactic protein-1; MIP-1a: macrophage inflammatory protein 1 alpha; MIP-1 $\beta$ : macrophage inflammatory protein 1 beta; MSR-1: 
macrophage scavenger receptor 1; NGF: nerve growth factor; PEG: polyethylene glycol; QD: quantum dot; RANTES: regulated upon activation: normal T-cell expressed: and secreted (also known as chemokine (C-C motif) ligand 5 (CCL5)); RIP: ribosome inactivating protein; VEGF: vascular endothelial growth factor; XPS: X-ray photoelectron spectroscopy.

\section{Acknowledgements}

We thank Gary Howard and Stephen Ordway for editorial assistance and Vivian Shen for technical assistance. This work was supported in part by a grant from the NIH (AG030207-A2) and a gift from the S. D. Bechtel, Jr. Foundation. The J. David Gladstone Institutes received support from a National Center for Research Resources Grant RR18928-01.

\section{Author details}

'Gladstone Institute of Neurological Disease, 1650 Owens St., San Francisco CA 94158, USA. ${ }^{2}$ Department of Neurology, University of California, San Francisco, 505 Parnassus Ave., San Francisco CA, 94143, USA. ${ }^{3}$ Department of Bioengineering and Therapeutic Sciences, University of California, San Francisco, 513 Parnassus Ave., San Francisco CA 94143, USA. ${ }^{4}$ Department of Chemistry, University of Pittsburgh, 219 Parkman Ave., Pittsburgh PA 15260, USA. ${ }^{5}$ Department of Mechanical Engineering, Colorado State University, Fort Collins CO 80523, USA

\section{Authors' contributions}

SSM, BS, TK, KP, MEW, and YZ conducted experiments. LG, LM, and TD conceived the project. LG, BS, MP and SSM designed experiments. LG and SSM wrote the manuscript. All authors have read and approved the final manuscript.

\section{Competing interests}

The authors declare that they have no competing interests.

Received: 21 October 2011 Accepted: 24 January 2012

Published: 24 January 2012

\section{References}

1. Frank-Cannon TC, Alto LT, McAlpine FE, Tansey MG: Does neuroinflammation fan the flame in neurodegenerative diseases? $\mathrm{Mol}$ Neurodegener 2009, 4:47.

2. Colton CA: Heterogeneity of microglial activation in the innate immune response in the brain. J Neuroimmune Pharmacol 2009, 4:399-418.

3. Akiyama $\mathrm{H}, \mathrm{McGeer} \mathrm{PL}$ : Brain microglia constitutively express $\beta-2$ integrins. J Neuroimmunol 1990, 30:81-93.

4. Town T, Nikolic V, Tan J: The microglial "activation" continuum: from innate to adaptive responses. J Neuroinflammation 2005, 2:24.

5. Lucin KM, Wyss-Coray T: Immune activation in brain aging and neurodegeneration: too much or too little? Neuron 2009, 64:110-122.

6. Block ML, Zecca L, Hong JS: Microglia-mediated neurotoxicity: uncovering the molecular mechanisms. Nat Rev Neurosci 2007, 8:57-69.

7. Bard F, Barbour R, Cannon C, Carretto R, Fox M, Games D, Guido T, Hoenow K, Hu K, Johnson-Wood K, et al: Epitope and isotype specificities of antibodies to $\beta$-amyloid peptide for protection against Alzheimer's disease-like neuropathology. Proc Natl Acad Sci USA 2003, 100:2023-2028.

8. Wyss-Coray T, Mucke L: Inflammation in neurodegenerative disease-a double-edged sword. Neuron 2002, 35:419-432.

9. Chen J, Zhou Y, Mueller-Steiner S, Chen LF, Kwon H, Yi S, Mucke L, Gan L: SIRT1 Protects against Microglia-dependent Amyloid-\{beta\} Toxicity through Inhibiting NF-\{kappa\}B Signaling. J Biol Chem 2005, 280:40364-40374.

10. Michalet X, Pinaud FF, Bentolila LA, Tsay JM, Doose S, Li JJ, Sundaresan G, Wu AM, Gambhir SS, Weiss S: Quantum dots for live cells, in vivo imaging, and diagnostics. Science 2005, 307:538-544.

11. Du W, Wang Y, Luo Q, Liu BF: Optical molecular imaging for systems biology: from molecule to organism. Anal Bioanal Chem 2006, 386:444-457.

12. Alivisatos AP, Gu W, Larabell C: Quantum dots as cellular probes. Annu Rev Biomed Eng 2005, 7:55-76.

13. Bruchez M Jr, Moronne M, Gin P, Weiss S, Alivisatos AP: Semiconductor nanocrystals as fluorescent biological labels. Science 1998 281:2013-2016.
14. Gao X, Cui Y, Levenson RM, Chung LW, Nie S: In vivo cancer targeting and imaging with semiconductor quantum dots. Nat Biotechnol 2004, 22:969-976.

15. Azzazy HM, Mansour MM, Kazmierczak SC: From diagnostics to therapy: prospects of quantum dots. Clin Biochem 2007, 40:917-927.

16. Jaiswal JK, Simon SM: Potentials and pitfalls of fluorescent quantum dots for biological imaging. Trends Cell Biol 2004, 14:497-504.

17. Vu TQ, Maddipati R, Blute TA, Nehilla BJ, Nusblat L, Desai TA: Peptideconjugated quantum dots activate neuronal receptors and initiate downstream signaling of neurite growth. Nano Lett 2005, 5:603-607.

18. Delehanty JB, Mattoussi H, Medintz IL: Delivering quantum dots into cells: strategies, progress and remaining issues. Anal Bioanal Chem 2009, 393:1091-1105.

19. Jackson H, Muhammad O, Daneshvar H, Nelms J, Popescu A, Vogelbaum MA, Bruchez M, Toms SA: Quantum dots are phagocytized by macrophages and colocalize with experimental gliomas. Neurosurgery 2007, 60:524-529, discussion 529-530.

20. Kauppinen TM, Swanson RA: Poly(ADP-ribose) polymerase-1 promotes microglial activation, proliferation, and matrix metalloproteinase-9mediated neuron death. J Immunol 2005, 174:2288-2296.

21. Smith PK, Krohn RI, Hermanson GT, Mallia AK, Gartner FH, Provenzano MD, Fujimoto EK, Goeke NM, Olson BJ, Klenk DC: Measurement of protein using bicinchoninic acid. Anal Biochem 1985, 150:76-85.

22. Doherty GJ, McMahon HT: Mechanisms of endocytosis. Annu Rev Biochem 2009, 78:857-902.

23. Wang $L H$, Rothberg KG, Anderson RG: Mis-assembly of clathrin lattices on endosomes reveals a regulatory switch for coated pit formation. J Cell Biol 1993, 123:1107-1117.

24. Sampath P, Pollard TD: Effects of cytochalasin, phalloidin, and pH on the elongation of actin filaments. Biochemistry 1991, 30:1973-1980.

25. Yarar D, Waterman-Storer CM, Schmid SL: A dynamic actin cytoskeleton functions at multiple stages of clathrin-mediated endocytosis. Mol Biol Cell 2005, 16:964-975.

26. Hussain KM, Leong KL, Ng MM, Chu JJ: The essential role of clathrinmediated endocytosis in the infectious entry of human enterovirus 71. $J$ Biol Chem 2011, 286:309-321.

27. Krieger M, Herz J: Structures and functions of multiligand lipoprotein receptors: macrophage scavenger receptors and LDL receptor-related protein (LRP). Annu Rev Biochem 1994, 63:601-637.

28. Salminen A, Ojala J, Kauppinen A, Kaarniranta K, Suuronen T: Inflammation in Alzheimer's disease: amyloid-beta oligomers trigger innate immunity defence via pattern recognition receptors. Prog Neurobiol 2009, 87:181-194.

29. Yang CN, Shiao YJ, Shie FS, Guo BS, Chen PH, Cho CY, Chen YJ, Huang FL, Tsay HJ: Mechanism mediating oligomeric Abeta clearance by naive primary microglia. Neurobiol Dis 2011, 42:221-230.

30. Marzolo MP, von Bernhardi R, Inestrosa NC: Mannose receptor is present in a functional state in rat microglial cells. J Neurosci Res 1999, 58:387-395.

31. Meda L, Baron P, Prat E, Scarpini E, Scarlato G, Cassatella MA, Rossi F: Proinflammatory profile of cytokine production by human monocytes and murine microglia stimulated with beta-amyloid[25-35]. $J$ Neuroimmunol 1999, 93:45-52.

32. El Khoury J, Hickman SE, Thomas CA, Cao L, Silverstein SC, Loike JD: Scavenger receptor-mediated adhesion of microglia to beta-amyloid fibrils. Nature 1996, 382:716-719.

33. Heldmann U, Mine Y, Kokaia Z, Ekdahl CT, Lindvall O: Selective depletion of Mac-1-expressing microglia in rat subventricular zone does not alter neurogenic response early after stroke. Exp Neurol 2011, 229:391-398.

34. Montero M, Gonzalez B, Zimmer J: Immunotoxic depletion of microglia in mouse hippocampal slice cultures enhances ischemia-like neurodegeneration. Brain Res 2009, 1291:140-152.

35. Mandrekar S, Jiang Q, Lee CY, Koenigsknecht-Talboo J, Holtzman DM, Landreth GE: Microglia mediate the clearance of soluble Abeta through fluid phase macropinocytosis. J Neurosci 2009, 29:4252-4262.

36. Boillee S, Yamanaka K, Lobsiger CS, Copeland NG, Jenkins NA, Kassiotis G, Kollias G, Cleveland DW: Onset and progression in inherited ALS determined by motor neurons and microglia. Science 2006, 312:1389-1392

37. Howell OW, Rundle JL, Garg A, Komada M, Brophy PJ, Reynolds R: Activated microglia mediate axoglial disruption that contributes to 
axonal injury in multiple sclerosis. J Neuropathol Exp Neurol 2010, 69:1017-1033.

38. Tansey MG, Goldberg MS: Neuroinflammation in Parkinson's disease: its role in neuronal death and implications for therapeutic intervention. Neurobiol Dis 2010, 37:510-518.

39. Hamza TH, Zabetian CP, Tenesa A, Laederach A, Montimurro J, Yearout D, Kay DM, Doheny KF, Paschall J, Pugh $E$, et al: Common genetic variation in the HLA region is associated with late-onset sporadic Parkinson's disease. Nat Genet 2010, 42:781-785.

40. Hardman R: A toxicologic review of quantum dots: toxicity depends on physicochemical and environmental factors. Environ Health Perspect 2006, 114:165-172.

41. Zhang T, Stilwell JL, Gerion D, Ding L, Elboudwarej O, Cooke PA, Gray JW, Alivisatos AP, Chen FF: Cellular effect of high doses of silica-coated quantum dot profiled with high throughput gene expression analysis and high content cellomics measurements. Nano Lett 2006, 6:800-808.

42. Clift MJ, Rothen-Rutishauser B, Brown DM, Duffin R, Donaldson K, Proudfoot L, Guy K, Stone V: The impact of different nanoparticle surface chemistry and size on uptake and toxicity in a murine macrophage cell line. Toxicol Appl Pharmacol 2008, 232:418-427.

43. Clift MJ, Varet J, Hankin SM, Brownlee B, Davidson AM, Brandenberger C Rothen-Rutishauser B, Brown DM, Stone V: Quantum dot cytotoxicity in vitro: An investigation into the cytotoxic effects of a series of different surface chemistries and their core/shell materials. Nanotoxicology 2011, 5:664-74.

44. Seleverstov O, Phang JM, Zabirnyk O: Semiconductor nanocrystals in autophagy research: methodology improvement at nanosized scale. Methods Enzymol 2009, 452:277-296.

45. Seleverstov O, Zabirnyk O, Zscharnack M, Bulavina L, Nowicki M, Heinrich JM, Yezhelyev M, Emmrich F, O'Regan R, Bader A: Quantum dots for human mesenchymal stem cells labeling. A size-dependent autophagy activation. Nano Lett 2006, 6:2826-2832.

46. Stern ST, Zolnik BS, McLeland CB, Clogston J, Zheng J, McNeil SE: Induction of autophagy in porcine kidney cells by quantum dots: a common cellular response to nanomaterials? Toxicol Sci 2008, 106:140-152.

47. Zabirnyk O, Yezhelyev M, Seleverstov O: Nanoparticles as a novel class of autophagy activators. Autophagy 2007, 3:278-281.

48. Pattingre S, Tassa A, Qu X, Garuti R, Liang XH, Mizushima N, Packer M, Schneider MD, Levine B: BCl-2 antiapoptotic proteins inhibit Beclin 1dependent autophagy. Cell 2005, 122:927-939.

doi:10.1186/1742-2094-9-22

Cite this article as: Minami et al: Selective targeting of microglia by quantum dots. Journal of Neuroinflammation 2012 9:22.

\section{Submit your next manuscript to BioMed Central and take full advantage of:}

- Convenient online submission

- Thorough peer review

- No space constraints or color figure charges

- Immediate publication on acceptance

- Inclusion in PubMed, CAS, Scopus and Google Scholar

- Research which is freely available for redistribution

Submit your manuscript at www.biomedcentral.com/submit
Biomed Central 